\title{
All-or-Nothing Phenomena: From Single-Letter to High Dimensions
}

\author{
Galen Reeves * Jiaming $\mathrm{Xu}^{\dagger} \quad$ Ilias Zadik ${ }^{\ddagger}$
}

January 1, 2020

\begin{abstract}
We consider the linear regression problem of estimating a $p$-dimensional vector $\beta$ from $n$ observations $Y=X \beta+W$, where $\beta_{j} \stackrel{\text { i.i.d. }}{\sim} \pi$ for a real-valued distribution $\pi$ with zero mean and unit variance, $X_{i j} \stackrel{\text { i.i.d. }}{\sim} \mathcal{N}(0,1)$, and $W_{i} \stackrel{\text { i.i.d. }}{\sim} \mathcal{N}\left(0, \sigma^{2}\right)$. In the asymptotic regime where $n / p \rightarrow \delta$ and $p / \sigma^{2} \rightarrow$ snr for two fixed constants $\delta$, snr $\in(0, \infty)$ as $p \rightarrow \infty$, the limiting (normalized) minimum mean-squared error (MMSE) has been characterized by the MMSE of an associated single-letter (additive Gaussian scalar) channel.

In this paper, we show that if the MMSE function of the single-letter channel converges to a step function, then the limiting MMSE of estimating $\beta$ in the linear regression problem converges to a step function which jumps from 1 to 0 at a critical threshold. Moreover, we establish that the limiting meansquared error of the (MSE-optimal) approximate message passing algorithm also converges to a step function with a larger threshold, providing evidence for the presence of a computational-statistical gap between the two thresholds.
\end{abstract}

\section{Introduction}

Consider the classical linear regression model

$$
Y=X \beta+W
$$

where $X \in \mathbb{R}^{n \times p}$ with $X_{i j} \stackrel{\text { i.i.d. }}{\sim} \mathcal{N}(0,1), \beta \in \mathbb{R}^{p}$ with $\beta_{j} \stackrel{\text { i.i.d. }}{\sim} \pi$ for a distribution $\pi$ with zero mean and unit variance, and $W \in \mathbb{R}^{n}$ with $W_{i} \stackrel{\text { i.i.d. }}{\sim} \mathcal{N}\left(0, \sigma^{2}\right)$. We are interested in estimating $\beta$ from observation of $(X, Y)$. For a given estimator $\widehat{\beta}(X, Y)$, the normalized mean squared-error of estimating $\beta$ is given by

$$
\operatorname{MSE}(\widehat{\beta}):=\frac{1}{p} \mathbb{E}\left[\|\beta-\widehat{\beta}\|^{2}\right] \text {. }
$$

Let MMSE denote the minimum of $\operatorname{MSE}(\widehat{\beta})$ among all possible estimators $\widehat{\beta}$, or equivalently,

$$
\operatorname{MMSE}:=\frac{1}{p} \mathbb{E}\left[\|\beta-\mathbb{E}[\beta \mid X, Y]\|^{2}\right] .
$$

\footnotetext{
${ }^{*}$ G. Reeves is with the Department of Electrical and Computer Engineering and the Department of Statistical Science, Duke University, Durham, NC 27708 USA; e-mail: galen.reeves@duke.edu. G. Reeves is is supported by NSF Grants CCF-1718494 and CCF-1750362.

${ }^{\dagger} \mathrm{J} . \mathrm{Xu}$ is with the Fuqua School of Business, Duke University, Durham, NC 27708 USA; e-mail: jiamingxu.868@duke.edu. J. Xu is is supported by NSF Grants IIS-1932630, CCF-1850743, and CCF-1856424.

${ }^{\ddagger}$ I. Zadik is with the Center for Data Science, New York University, New York, NY 10011 USA; e-mail: zadik@nyu.edu. I. Zadik is supported by a CDS-Moore-Sloan postdoctoral fellowship.

This paper was presented at the 2019 IEEE International Workshop on Computational Advances in Multi-Sensor Adaptive Processing (CAMSAP), Guadeloupe, French West Indies.
} 
In this paper, we focus on the asymptotic regime:

$$
\frac{n}{p} \rightarrow \delta \quad \text { and } \quad \frac{p}{\sigma^{2}} \rightarrow \mathrm{snr}, \quad \text { as } p \rightarrow \infty,
$$

for two fixed constants $\delta, \operatorname{snr} \in(0, \infty)$. Note that $\delta$ is the under-sampling ratio and snr is the signal-to-noise ratio in view of $\mathbb{E}\left[\|X \beta\|^{2}\right] / \mathbb{E}\left[\|W\|^{2}\right]=p / \sigma^{2}$.

Recent work [1] proves that under certain structural assumptions in terms of $(\pi, \delta$, snr), the limiting MMSE in the asymptotic regime (3) is characterized by the replica-symmetric (RS) formula through a single letter channel

$$
y=\sqrt{s} \beta_{0}+N,
$$

where $s>0, \beta_{0} \sim \pi$ and $N \sim \mathcal{N}(0,1)$ are independent. However, often the RS formula is too complicated to extract structural behavior of the limiting MMSE.

In this work, we propose generic conditions under which the limiting MMSE exhibits an all-or-nothing phenomena. More precisely, consider a family $\left(\pi_{\epsilon}, \delta_{\epsilon}, \mathrm{snr}_{\epsilon}\right)$ indexed by a positive parameter $\epsilon$ where $\pi_{\epsilon}$ has finite entropy $H_{\epsilon}:=H\left(\pi_{\epsilon}\right)$. We show that if the MMSE of the single letter channel (4) as a function of $s$ converges to a step function as $\epsilon \rightarrow 0$, then the limiting MMSE of the linear regression model (11) also converges to a step function, which jumps from 1 to 0 at a critical threshold $\delta_{\epsilon}=\delta_{\epsilon, \mathrm{MMSE}}$, where

$$
\delta_{\epsilon, \mathrm{MMSE}}:=\frac{2 H_{\epsilon}}{\log \left(1+\mathrm{snr}_{\epsilon}\right)} .
$$

In other words, an all-or-nothing phenomena in the single letter channel implies an all-or-nothing phenomena in the high-dimensional linear regression model. Moreover, we establish that the limiting MSE of the (MSEoptimal) approximate message passing (AMP) algorithm also converges to a step function, which jumps from 1 to 0 at a larger threshold $\delta_{\epsilon}=\delta_{\epsilon, \mathrm{AMP}}$, where

$$
\delta_{\epsilon, \mathrm{AMP}}:=\frac{2 H_{\epsilon}\left(1+\mathrm{snr}_{\epsilon}\right)}{\operatorname{snr}_{\epsilon}} .
$$

An important application of our general result is the binary linear regression model where $\beta_{j} \stackrel{\text { i.i.d. }}{\sim} \operatorname{Bern}(\epsilon)$. In this case, we show that the MMSE function of the single letter channel converges to a step function as the sparsity $\epsilon \rightarrow 0$. Then we obtain from our general result that the limiting MMSE of the binary linear regression model converges to a step function which jumps from 1 to 0 at the critical threshold $\delta_{\epsilon}=\frac{2 \epsilon \log (1 / \epsilon)}{\log \left(1+\operatorname{snr}_{\epsilon}\right)}$. This coincides with the all-or-nothing phenomenon established in [4 for the binary linear regression model where $\beta$ is chosen uniformly at random from the set of binary $k$-sparse vectors, in the highly sparse and high signal-to-noise ratio regime where $k / \sqrt{p} \rightarrow 0$ and $k / \sigma^{2}$ is above a sufficiently large constant. Furthermore, we deduce from our general result that the limiting MSE of the (MSE-optimal) AMP converges to a step function which jumps from 1 to 0 at the critical threshold $\delta_{\epsilon}=\frac{2 \epsilon \log (1 / \epsilon)\left(1+\mathrm{snr}_{\epsilon}\right)}{\mathrm{snr}_{\epsilon}}$. This coincides with the computational threshold for a number of computationally efficient methods in the literature such as LASSO or Orthogonal Matching Pursuit. In particular, our result adds to the existing evidence for the presence of a computational-statistical gap between the two thresholds (see [5,6] for an extended discussion and literature review on the presence of this computational-statistical gap).

\section{Preliminaries}

\subsection{The Replica Symmetric Formulas}

To describe the RS formulas, we first define the mutual information and MMSE functions for the single letter channel (4):

$$
I(s):=I\left(\beta_{0} ; \sqrt{s} \beta_{0}+N\right), \quad s>0
$$




$$
M(s):=\operatorname{mmse}\left(\beta_{0} \mid \sqrt{s} \beta_{0}+N\right), \quad s>0
$$

where $\beta_{0} \sim \pi$ and $N \sim \mathcal{N}(0,1)$ are independent. Both of these functions are non-negative and the unit variance assumption on $\pi$ means that for any $s>0$, (see [7] for details)

$$
\begin{aligned}
I(s) & \leq \frac{1}{2} \log (1+s) \leq \frac{s}{2}, \\
M(s) & \leq \frac{1}{1+s} \leq 1
\end{aligned}
$$

Moreover, the I-MMSE relation for the single-letter channel [7] states the derivative of the mutual information is one half the MMSE, that is $I^{\prime}(s)=\frac{1}{2} M(s)$.

Next, we define the potential function $\mathcal{F}:[0, \infty) \rightarrow[0, \infty)$ according to

$$
\mathcal{F}(s):=I(s)+\frac{\delta}{2} \phi\left(\frac{s}{\delta \mathrm{snr}}\right),
$$

where $\phi(x)=x-\log x-1$, and $\delta$, snr are respectively the undersampling ratio and the signal-to-noise ratio of our original model. Note that $\phi(x)$ is convex and non-negative on $(0, \infty)$.

Lemma 1. All stationary points of $\mathcal{F}(s)$ lie on the open interval between $\delta \mathrm{snr} /(1+\mathrm{snr})$ and $\delta \mathrm{snr}$.

Proof. By differentiation with respect to $s$ and the I-MMSE relation for the single-letter channel [7], we have that for any $s>0$

$$
\mathcal{F}^{\prime}(s) \propto M(s)+1 / \mathrm{snr}-\delta / s .
$$

The fact that $M(s)>0$ for all $s$ implies that $\mathcal{F}^{\prime}(s)$ is strictly positive for all $s \geq \delta$ snr, and thus $\mathcal{F}(s)$ is strictly increasing on $[\delta \mathrm{snr}, \infty)$. Alternatively, the fact that $M(s)<1$ for all $s>0$ implies that $\mathcal{F}^{\prime}(s)$ is strictly negative for all $s \leq \delta \mathrm{snr} /(1+\mathrm{snr})$, and thus $\mathcal{F}(s)$ is strictly decreasing on $(0, \delta \mathrm{snr} /(1+\mathrm{snr})]$.

In view of Lemma 1, the minimum of the potential function and the smallest and largest minimizers can be defined as follows:

$$
\begin{aligned}
\mathcal{F}^{*} & :=\min _{s} \mathcal{F}(s), \\
\underline{s}^{*} & :=\min \left\{s: \mathcal{F}(s)=\mathcal{F}^{*}\right\}, \\
\bar{s}^{*} & :=\max \left\{s: \mathcal{F}(s)=\mathcal{F}^{*}\right\} .
\end{aligned}
$$

Note that $\underline{s}^{*}=\bar{s}^{*}$ if and only if the minimum is attained at a unique point.

Proposition 2 (RS MMSE 2,3] ). For any $(\delta, \mathrm{snr}, \pi)$ for which (snr, $\pi$ ) satisfies the single-crossing property [2] and $\pi$ has finite fourth moment 1, the mutual information and MMSE satisfy

$$
\begin{aligned}
& \lim _{p \rightarrow \infty} \frac{1}{p} I(\beta ; X, Y)=\mathcal{F}^{*}, \\
& \limsup _{p \rightarrow \infty} \frac{1}{p} \mathbb{E}\left[\|\beta-\mathbb{E}[\beta \mid y, X]\|^{2}\right] \leq M\left(\bar{s}^{*}\right), \\
& \liminf _{p \rightarrow \infty} \frac{1}{p} \mathbb{E}\left[\|\beta-\mathbb{E}[\beta \mid y, X]\|^{2}\right] \geq M\left(\underline{s}^{*}\right),
\end{aligned}
$$

where the limits are taken as $\left(n=n_{p}, p, \sigma^{2}=\sigma_{p}^{2}\right)$ scale to infinity with $p \rightarrow+\infty, n / p \rightarrow \delta$ and $p / \sigma^{2} \rightarrow$ snr.

\footnotetext{
${ }^{1}$ A different set of assumptions on $(\delta$, snr, $\pi)$ for which the Proposition 2 holds can be found in [3,8]
} 
Next, we turn to the family of approximate message passing (AMP) [9, 10, algorithms and specifically to the case of MMSE-AMP which is proven in to be optimal among AMP algorithms in minimizing the MSE of the recovery problem of interest [11. For simplicity from now on when we say AMP we refer to the MMSE-AMP algorithm. It turns out that a related formula to the one given in Proposition 2 describes the asymptotic MSE associated with AMP.

The smallest stationary point is defined as

$$
s^{\mathrm{AMP}}:=\inf \left\{s: \mathcal{F}^{\prime}(s)=0\right\} .
$$

It is rather straightforward to check that $s^{\mathrm{AMP}}$ is attained by some positive value $s$ and therefore it is a stationary point of $\mathcal{F}(s)$. In particular, by Lemma 1 we have $s^{\mathrm{AMP}} \in(\delta \mathrm{snr} /(1+\mathrm{snr}), \delta \mathrm{snr})$.

For the next result, for $T \in \mathbb{N}$ let $\widehat{\beta}_{\mathrm{AMP}, T}(Y, X)$ be the output of the AMP estimator [11, Section II.C] with input data $(Y, X)$ after $T$ iterations.

Proposition 3 (AMP, [10,11]). For any $(\delta, \mathrm{snr}, \pi)$ where $\pi$ has a finite fourth moment, AMP satisfies

$$
\lim _{T \rightarrow+\infty} \lim _{p \rightarrow+\infty} \frac{1}{p} \mathbb{E}\left[\left\|\beta-\widehat{\beta}_{\mathrm{AMP}, T}(Y, X)\right\|^{2}\right]=M\left(s^{\mathrm{AMP}}\right)
$$

where the inner limit is taken as $\left(n=n_{p}, p, \sigma^{2}=\sigma_{p}^{2}\right)$ scale to infinity with $p \rightarrow+\infty, n / p \rightarrow \delta$ and $p / \sigma^{2} \rightarrow$ snr.

Remark 1. The results stated above imply that AMP is optimal whenever $\underline{s}^{*}=\bar{s}^{*}=s^{\mathrm{AMP}}$.

Remark 2. For a proof of Proposition 3 we refer the reader to the statement and proof of [11, Theorem 6].

\section{Main Results}

Let us consider now a family of coefficient distributions $\left(\pi_{\epsilon}\right)_{\epsilon>0}$ indexed by a positive-valued parameter $\epsilon>0$. We assume throughout the section that for each $\epsilon>0$ the distributions $\pi_{\epsilon}$ has zero mean, unit variance and finite entropy $H_{\epsilon}$. Our results are all based on the following assumption on the family $\pi_{\epsilon}$.

Assumption 1. Let $\left(\pi_{\epsilon}\right)_{\epsilon>0}$ be a family of distributions with unit variance and finite entropy $H_{\epsilon}$. The MMSE function $M_{\epsilon}(s)$ of the single letter channel, as defined in (8), for $\pi_{\epsilon}$ coefficient distribution is assumed to converge pointwise to a step function as $\epsilon \rightarrow 0$ in the following sense

$$
\lim _{\epsilon \rightarrow 0} M_{\epsilon}\left(2 H_{\epsilon} t\right)= \begin{cases}1, & t \in[0,1) \\ 0, & t \in(1, \infty) .\end{cases}
$$

Remark 3. It can be straightforwardly checked using the I-MMSE relation for the single-letter channel [7] that the rescaling in the argument of $M_{\epsilon}$ by twice the entropy term, i.e. by $2 H_{\epsilon}$, is necessary for the convergence of $M_{\epsilon}$ to the step function. To see why, observe that $M_{\epsilon}(s)$, using the I-MMSE relation, satisfies the integral constraint $\int_{0}^{\infty} M_{\epsilon}\left(2 H_{\epsilon} t\right) d t=1$. Thus, convergence to a step function at a threshold other than $2 H_{\epsilon}$ would violate this constraint.

Remark 4. As we establish later in the section, Assumption 1 is satisfied for the family of (normalized) Bernoulli distributions with probability $\epsilon$. See Fig. 1 for a graphical illustration. We expect the assumption to hold under greater generality. 1

We now present our two main results assuming the family of distributions $\left(\pi_{\epsilon}\right)_{\epsilon>0}$ satisfies Assumption

Theorem 4. Let $\left(\pi_{\epsilon}\right)_{\epsilon>0}$ satisfy Assumption 1. Given a number $r \in(0,1) \cup(1, \infty)$, let $\left(\delta_{\epsilon}, \mathrm{snr}_{\epsilon}, \pi_{\epsilon}\right)_{\epsilon>0}$ be a family of triplets such that

$$
\lim _{\epsilon \rightarrow 0} \frac{\delta_{\epsilon}}{\delta_{\epsilon, \mathrm{MMSE}}}=r
$$


$I_{\epsilon}\left(2 H_{\epsilon} t\right) / H_{\epsilon}$

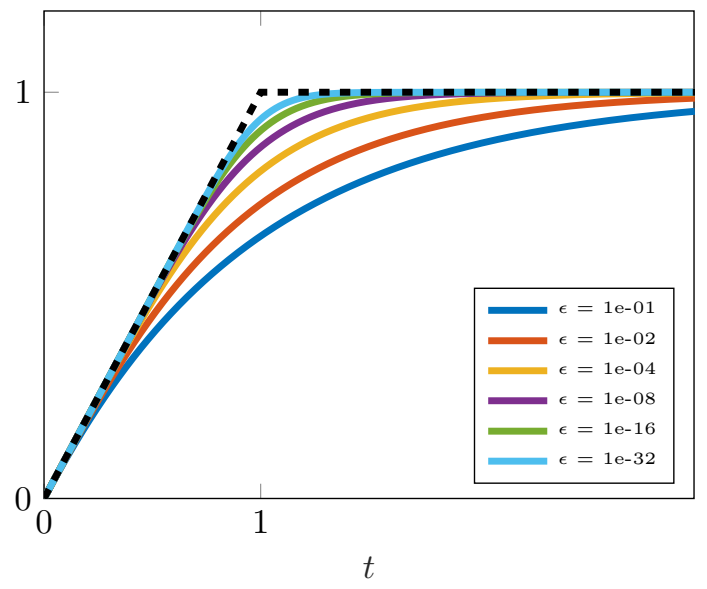

$M_{\epsilon}\left(2 H_{\epsilon} t\right)$

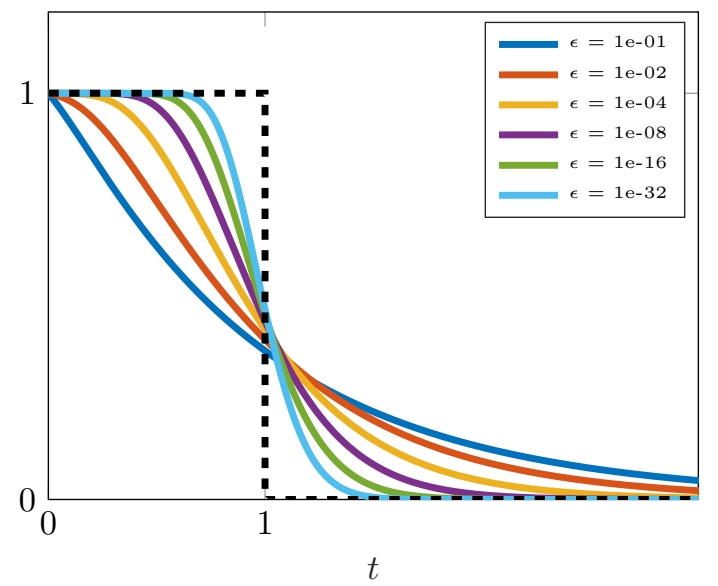

Figure 1: Single letter mutual information and MMSE functions corresponding to the Bernoulli( $\epsilon)$ distribution normalized to unit variance.

Then, the minimizers of the RS potential function exhibit the all-or-nothing behavior in the small- $\epsilon$ limit depending on whether $r$ is greater than or less than one:

$$
\begin{array}{ccc}
r \in(0,1) & \Longrightarrow & \lim _{\epsilon \rightarrow 0} M_{\epsilon}\left(\bar{s}_{\epsilon}^{*}\right)=1 \\
r \in(1, \infty) & \Longrightarrow & \lim _{\epsilon \rightarrow 0} M_{\epsilon}\left(\underline{s}_{\epsilon}^{*}\right)=0 .
\end{array}
$$

Combining Theorem 4 with Proposition 2 we obtain the following Corollary.

Corollary 5 (All-or-nothing MMSE behavior). Let $r \in(0,1) \cup(1, \infty)$. For any family of triplets $\left(\delta_{\epsilon}, \mathrm{snr}_{\epsilon}, \pi_{\epsilon}\right)_{\epsilon>0}$, suppose that for any $\epsilon>0,\left(\mathrm{snr}_{\epsilon}, \pi_{\epsilon}\right)$ satisfies the single-crossing property, $\pi_{\epsilon}$ has finite fourth moment, $\left(\pi_{\epsilon}\right)_{\epsilon>0}$ satisfies Assumption [1, and (21) holds. Then it holds that

$$
\lim _{\epsilon \rightarrow 0} \lim _{p \rightarrow \infty} \frac{1}{p} \mathbb{E}\left[\|\beta-\mathbb{E}[\beta \mid X, Y]\|^{2}\right]= \begin{cases}1, & r \in[0,1) \\ 0, & r \in(1, \infty) .\end{cases}
$$

where the inner limits are taken as $\left(n=n_{p}, p, \sigma^{2}=\sigma_{p}^{2}\right)$ scale to infinity with $p \rightarrow+\infty, n / p \rightarrow \delta_{\epsilon}$ and $p / \sigma^{2} \rightarrow$ snr $_{\epsilon}$.

We next present our second main result.

Theorem 6. Let $\left(\pi_{\epsilon}\right)_{\epsilon>0}$ satisfy Assumption 1. Given a number $r \in(0,1) \cup(1, \infty)$, let $\left(\delta_{\epsilon}, \operatorname{snr}_{\epsilon}, \pi_{\epsilon}\right)$ be such that

$$
\lim _{\epsilon \rightarrow 0} \frac{\delta_{\epsilon}}{\delta_{\epsilon, \mathrm{AMP}}}=r
$$

Then, the smallest stationary point $s^{\mathrm{AMP}}$ exhibits the all-or-nothing behavior in the small- $\epsilon$ limit depending on whether $r$ is greater than or less than one:

$$
\begin{gathered}
r \in(0,1) \quad \Longrightarrow \quad \lim _{\epsilon \rightarrow 0} M_{\epsilon}\left(s_{\epsilon}^{\mathrm{AMP}}\right)=1 \\
r \in(1, \infty) \quad \Longrightarrow \quad \lim _{\epsilon \rightarrow 0} M_{\epsilon}\left(s_{\epsilon}^{\mathrm{AMP}}\right)=0 .
\end{gathered}
$$


For the result, for $T \in \mathbb{N}$ let $\widehat{\beta}_{\mathrm{AMP}, T}(Y, X)$ the output of the AMP estimator [11, Section II.C] with input data $(X, Y)$ after $T$ iterations. Combining Theorem 6 with Proposition 3 we obtain the following Corollary on the performance of AMP.

Corollary 7 (All-or-nothing AMP behavior). Let $r \in(0,1) \cup(1, \infty)$. For any family of triplets $\left(\delta_{\epsilon}, \mathrm{snr}_{\epsilon}, \pi_{\epsilon}\right)_{\epsilon>0}$, suppose that each $\pi_{\epsilon}$ has a finite fourth moment, the family $\left(\pi_{\epsilon}\right)_{\epsilon>0}$ satisfies Assumption [1, and (25) holds. Then it holds that

$$
\lim _{\epsilon \rightarrow 0} \lim _{T \rightarrow+\infty} \lim _{p \rightarrow \infty} \frac{1}{p} \mathbb{E}\left[\left\|\beta-\widehat{\beta}_{\mathrm{AMP}, T}(X, Y)\right\|^{2}\right]= \begin{cases}1, & r \in[0,1) \\ 0, & r \in(1, \infty) .\end{cases}
$$

where the inner limits are taken as $\left(n=n_{p}, p, \sigma^{2}=\sigma_{p}^{2}\right)$ scale to infinity with $p \rightarrow+\infty, n / p \rightarrow \delta_{\epsilon}$ and $p / \sigma^{2} \rightarrow \operatorname{snr}_{\epsilon}$.

\subsection{Illustration of Theorems 4 and 6 via normalized potential function}

To provide insight into the behavior described by Theorems 4 and 6 , we consider the scaling $\delta_{\epsilon}=2 r H_{\epsilon} / \log (1+$ snr) where the pair (snr, $r$ ) is considered fixed with respect to $\epsilon>0$. Note that in this scaling, $\delta_{\epsilon}=r \delta_{\epsilon, \mathrm{MmSE}}$. Define the normalized potential function

$$
\widetilde{\mathcal{F}}_{\epsilon, r}(t):=\frac{\mathcal{F}\left(2 H_{\epsilon} t\right)}{H_{\epsilon}}=\frac{I_{\epsilon}\left(2 H_{\epsilon} t\right)}{H_{\epsilon}}+\frac{r}{\log (1+\mathrm{snr})} \phi\left(\frac{t \log (1+\mathrm{snr})}{r \mathrm{snr}}\right) .
$$

Note that only the first term depends on $\epsilon$. Under Assumption 1 using the I-MMSE relation we have $I_{\epsilon}\left(2 H_{\epsilon} t\right) / H_{\epsilon} \rightarrow 1 \wedge t$ as $\epsilon \rightarrow 0$. Thus for all $t>0$,

$$
\lim _{\epsilon \rightarrow 0} \widetilde{\mathcal{F}}_{\epsilon, r}(t)=\widetilde{\mathcal{F}}_{0, r}(t)
$$

where

$$
\widetilde{\mathcal{F}}_{0, r}(t):=1 \wedge t+\frac{r}{\log (1+\mathrm{snr})} \phi\left(\frac{t \log (1+\mathrm{snr})}{r \mathrm{snr}}\right) .
$$

For $r \in(0,1) \cup(1, \infty)$, the function $\widetilde{\mathcal{F}}_{0, r}(t)$ has a unique global minimizer given by

$$
t^{*}= \begin{cases}\frac{r \mathrm{snr}}{(1+\mathrm{snr}) \log (1+\mathrm{snr})}, & r \in(0,1) \\ \frac{r \mathrm{snr}}{\log (1+\mathrm{snr})} & r \in(1, \infty) .\end{cases}
$$

Importantly, using the elementary inequality $x /(x+1) \leq \log (1+x) \leq x$ for all $x>-1$, we deduce

$$
\begin{array}{cc}
r \in(0,1) & \Longrightarrow \quad t^{*} \in(0,1) \\
r \in(1, \infty) & \Longrightarrow \quad t^{*} \in(1, \infty) .
\end{array}
$$

This dichotomy together with Assumption 1 and equality (30) suggest the following all-or-nothing behavior; as $\epsilon \rightarrow 0$, when $r<1$ the MMSE converges to one and when $r>1$ the MMSE converges to zero. This is the same all-or-nothing behavior described and rigorously established in Theorem 4 Furthermore, the smallest stationary point $t^{\mathrm{AMP}}$ is given by

$$
t^{\mathrm{AMP}}= \begin{cases}\frac{r \mathrm{snr}}{(1+\mathrm{snr}) \log (1+\mathrm{snr})}, & r \in\left(0, r^{\mathrm{AMP}}\right) \\ \frac{r \mathrm{snr}}{\log (1+\mathrm{snr})}, & r \in\left(r^{\mathrm{AMP}}, \infty\right),\end{cases}
$$


where $r^{\mathrm{AMP}}:=\delta_{\epsilon, \mathrm{AMP}} / \delta_{\epsilon, \mathrm{MMSE}}=(1+1 / \mathrm{snr}) \log (1+\mathrm{snr})$. Similar to above, we deduce

$$
\begin{aligned}
r \in\left(0, r^{\mathrm{AMP}}\right) & \Longrightarrow t^{\mathrm{AMP}} \in(0,1) \\
r \in\left(r^{\mathrm{AMP}}, \infty\right) & \Longrightarrow t^{\mathrm{AMP}} \in(1, \infty) .
\end{aligned}
$$

This dichotomy together with Assumption 1 and equality (30), suggest the following all-or-nothing behavior; as $\epsilon \rightarrow 0$, when $r<r^{\text {AMP }}$ the MSE of the AMP converges to one and when $r>r^{\text {AMP }}$ the MSE of the AMP converges to zero. This is the same all-or-nothing behavior described and rigorously established in Theorem 6 .

This behavior of the normalized potential function is illustrated graphically in Figure 2 where both $\widetilde{\mathcal{F}}_{\epsilon, r}(t)$ and $\widetilde{\mathcal{F}}_{0, r}(t)$ are plotted as a function of $t$ for various $r$.

\section{Application: Sparse binary regression}

We now present our main application of our two results to sparse binary regression, where $\beta_{i} \stackrel{\text { i.i.d. }}{\sim} \operatorname{Bern}(\epsilon)$. To this end, we first consider the case where $\beta_{i}$ is i.i.d. drawn from the following two-point distribution:

$$
\pi_{\epsilon}=(1-\epsilon) \delta_{\mu_{1}}+\epsilon \delta_{\mu_{2}}
$$

where $\delta_{x}$ denotes a Dirac distribution with mass at $x \in \mathbb{R}$, and $\mu_{1}=-\sqrt{\epsilon /(1-\epsilon)}$ and $\mu_{2}=\sqrt{(1-\epsilon) / \epsilon}$ are chosen such that $\pi_{\epsilon}$ has zero mean and unit variance. The following Lemma holds for the family of MMSE functions $\left(M_{\epsilon}(s)\right)_{\epsilon>0}$ :

Lemma 8. The distribution $\pi_{\epsilon}$ in (34) has entropy $H_{\epsilon}=-\epsilon \log \epsilon-(1-\epsilon) \log (1-\epsilon)$ and MMSE function

$$
M_{\epsilon}(s)=\mathbb{E}\left[\frac{1}{1-\epsilon+\epsilon \exp \left(\frac{s}{2 \epsilon(1-\epsilon)}+\sqrt{\frac{s}{\epsilon(1-\epsilon)}} N\right)}\right],
$$

where $N \sim \mathcal{N}(0,1)$. Furthermore, the distribution $\pi_{\epsilon}$ satisfies the single-crossing condition [2] for all snr $>0$ and

$$
\begin{array}{r}
\lim _{\epsilon \rightarrow 0} H_{\epsilon} /(\epsilon \log 1 / \epsilon)=1 \\
\limsup _{\epsilon \rightarrow 0}\left|M_{s>0}(s)-Q\left(\frac{s-2 \epsilon \log (1 / \epsilon)}{2 \sqrt{s \epsilon}}\right)\right|=0,
\end{array}
$$

where $Q(z)=\int_{z}^{\infty}(2 \pi)^{-1 / 2} \exp \left(-t^{2} / 2\right) d t$.

An immediate implication of the result is that the family of distributions $\left(\pi_{\epsilon}\right)_{\epsilon>0}$ satisfies Assumption [1 as well as the conditions of Corollaries 5 and 7 Hence, all-or-nothing phase transitions hold for the limiting MMSE around $\delta_{\epsilon, \mathrm{MMSE}}$ and for the MSE of the AMP around $\delta_{\epsilon, \mathrm{AMP}}$. Using that $\lim _{\epsilon \rightarrow 0} H_{\epsilon} /(\epsilon \log 1 / \epsilon)=1$, we can further simplify the phase transition points given in (5), (6) by observing

$$
\lim _{\epsilon \rightarrow 0} \delta_{\epsilon, \mathrm{MMSE}} /\left(\frac{2 \epsilon \log (1 / \epsilon)}{\log \left(1+\operatorname{snr}_{\epsilon}\right)}\right)=1
$$

and

$$
\lim _{\epsilon \rightarrow 0} \delta_{\epsilon, \mathrm{AMP}} /\left(\frac{2\left(1+\mathrm{snr}_{\epsilon}\right) \epsilon \log (1 / \epsilon)}{\mathrm{snr}_{\epsilon}}\right)=1
$$


normalized potential function $\widetilde{\mathcal{F}}_{\epsilon, r}(t)$ with snr $=5$ and $\epsilon=10^{-16}$

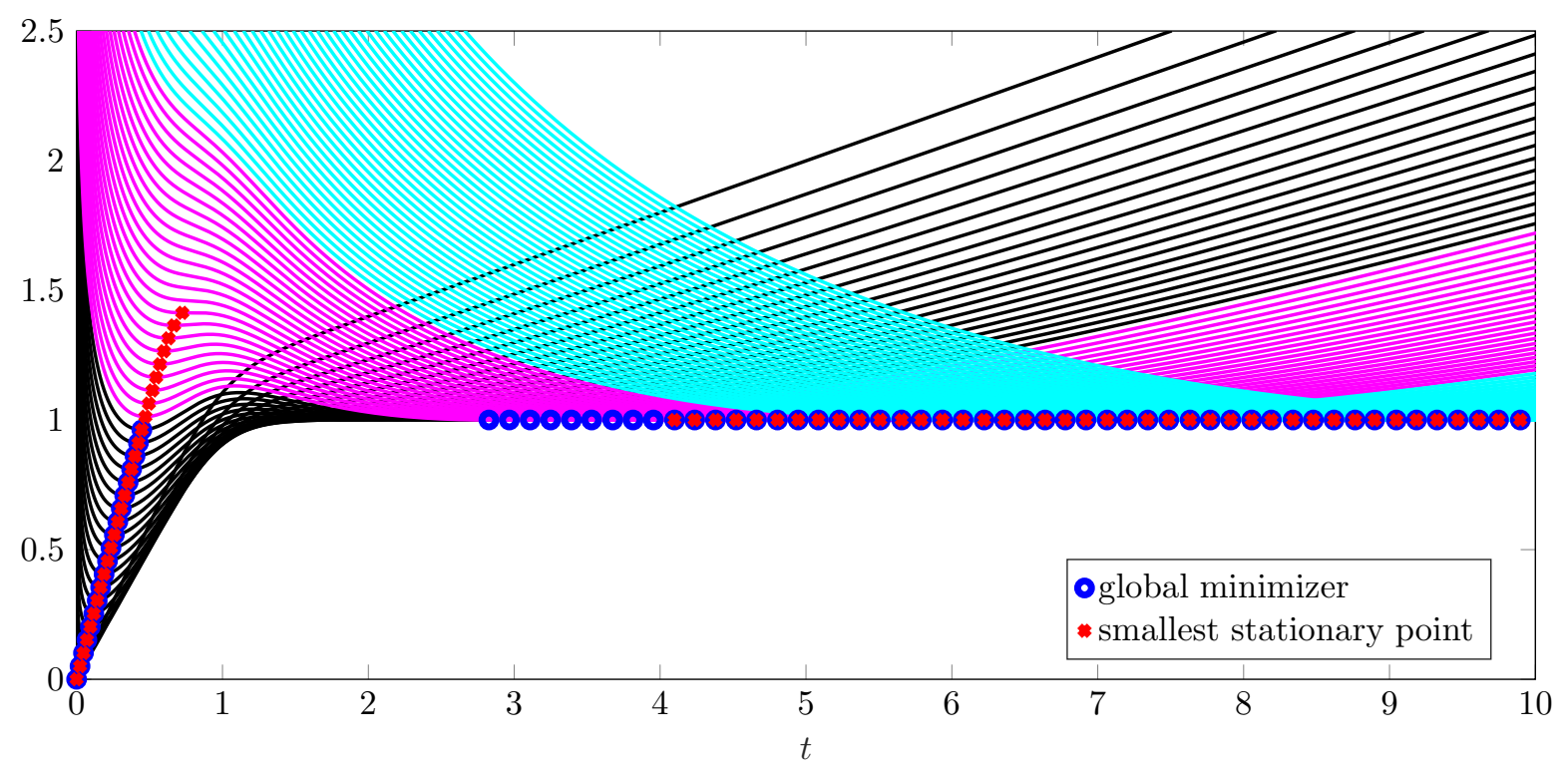

normalized potential function $\widetilde{\mathcal{F}}_{0, r}(t)$ with snr $=5$

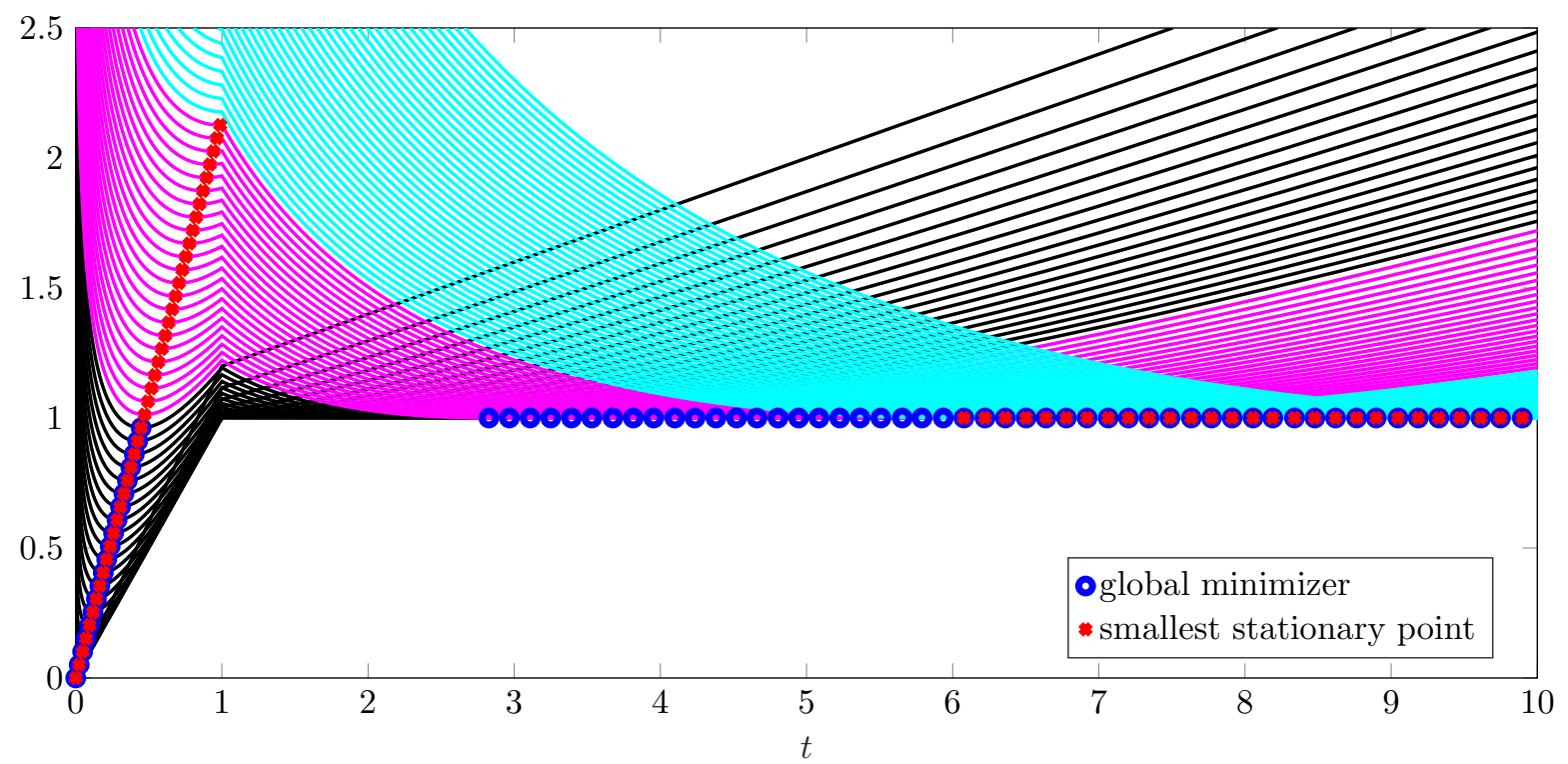

Figure 2: Illustration of the normalized potential functions, $\widetilde{\mathcal{F}}_{\epsilon, r}(t)$ for $\epsilon=10^{-16}$ (top plot) and $\widetilde{\mathcal{F}}_{0, r}(t)$ (bottom plot), where snr $=5$ and $r$ varies in $(0,+\infty)$. Black-colored curves correspond to $r \in(0,1)$, magenta-colored curves correspond to $r \in\left(1, r^{\mathrm{AMP}}\right)$ and cyan-colored curves correspond to $r \in\left(r^{\mathrm{AMP}}, \infty\right)$. Note that the global minimizer of $\widetilde{\mathcal{F}}_{0, r}(t)$ transitions from being less than one to bigger than one exactly at $r=1$, while the smallest stationary point transitions from being less than one to bigger than one exactly at $r=r^{\text {AMP }}$. A similar approximate behavior takes place for $\widetilde{\mathcal{F}}_{\epsilon, r}(t)$ with $\epsilon=10^{-16}$. 
Next, we extend the above results to the sparse binary regression problem of interest, where $\beta_{i} \stackrel{\text { i.i.d. }}{\sim} \operatorname{Bern}(\epsilon)$. We denote by $k=\epsilon p$ the (expected) number of non-zero coordinates of $\beta$. Define

$$
\widetilde{\beta}=\frac{\beta-\mathbb{E}[\beta]}{\sqrt{\epsilon(1-\epsilon)}} .
$$

Then $\widetilde{\beta}_{i}^{\text {i.i.d. }} \pi_{\epsilon}$ as given in (34). Moreover, define

$$
\widetilde{Y}=\frac{Y-X \mathbb{E}[\beta]}{\sqrt{\epsilon(1-\epsilon)}}, \quad \widetilde{W}=\frac{W}{\sqrt{\epsilon(1-\epsilon)}} .
$$

Then it follows that $\widetilde{Y}=X \widetilde{\beta}+\widetilde{W}$. Since $\widetilde{W}_{i}^{\text {i.i.d. }} \mathcal{N}\left(0, \widetilde{\sigma}^{2}\right)$ with $\widetilde{\sigma}=\sigma / \sqrt{\epsilon(1-\epsilon)}$, it follows that

$$
\operatorname{snr}_{\epsilon}=\frac{p}{\widetilde{\sigma}^{2}}=\frac{p \epsilon(1-\epsilon)}{\sigma^{2}} .
$$

Hence, according to Corollary [5, (38), (40), we obtain that the limiting MMSE exhibits an all-or-nothing behavior at

$$
\delta_{\epsilon, \mathrm{MMSE}}=2 \epsilon \log (1 / \epsilon) / \log \left(1+\epsilon(1-\epsilon) p / \sigma^{2}\right),
$$

which using $k=\epsilon p$ as $\epsilon \rightarrow 0$ simplifies with negligible multiplicative error to

$$
\delta_{\epsilon, \mathrm{MMSE}}=2(k / p) \log (p / k) / \log \left(1+k / \sigma^{2}\right) .
$$

Note that this is the exact information-theoretic threshold for which an all-or-nothing phenomenon has been proven to hold when $\lim \sup _{p} \log k / \log p<0.5$ in [4].

Similarly, according to Corollary 7. (39), and (40), the limiting MSE of the AMP exhibits an all-or-nothing behavior at:

$$
\delta_{\epsilon, \mathrm{AMP}}=2\left(1+\frac{\sigma^{2}}{p \epsilon(1-\epsilon)}\right) \epsilon \log (1 / \epsilon),
$$

which using $k=\epsilon p$ as $\epsilon \rightarrow 0$ simplifies with negligible multiplicative error to

$$
\delta_{\epsilon, \mathrm{AMP}}=2\left(k+\sigma^{2}\right) \log (p / k) / p .
$$

Note that this is the exact computational threshold for a number of computationally efficient methods in the literature such as LASSO or Orthogonal Matching Pursuit (see [5,6] for references). Our result suggests that the threshold corresponds to a barrier also for AMP in a strong sense.

\section{References}

[1] Dongning Guo and S. Verdu, "Randomly spread cdma: asymptotics via statistical physics," IEEE Transactions on Information Theory, vol. 51, no. 6, pp. 1983-2010, June 2005.

[2] G. Reeves and H. D. Pfister, "The replica-symmetric prediction for random linear estimation with Gaussian matrices is exact," IEEE Trans. Inform. Theory, vol. 65, no. 4, pp. 2252-2283, Apr. 2019.

[3] J. Barbier, M. Dia, N. Macris, and F. Krzakala, "The mutual information in random linear estimation," in Proc. Annual Allerton Conf. on Commun., Control, and Comp., Monticello, IL, 2016.

[4] G. Reeves, J. Xu, and I. Zadik, "The all-or-nothing phenomenon in sparse linear regression," in Conference On Learning Theory (COLT), 2019, [Online]. Available https://arxiv.org/abs/1903.05046

[5] D. Gamarnik and I. Zadik, "High dimensional regression with binary coefficients. estimating squared error and a phase transtition," in COLT, 2017. 
[6] — - "Sparse high-dimensional linear regression. algorithmic barriers and a local search algorithm," 2017, [Online]. Available https://arxiv.org/abs/1711.04952.

[7] Dongning Guo, S. Shamai, and S. Verdu, "Mutual information and mmse in gaussian channels," in International Symposium onInformation Theory, 2004. ISIT 2004. Proceedings., June 2004, pp. 349349.

[8] J. Barbier, F. Krzakala, N. Macris, L. Miolane, and L. Zdeborová, "Optimal errors and phase transitions in high-dimensional generalized linear models," Proceedings of the National Academy of Sciences, vol. 116, no. 12, pp. 5451-5460, 2019. [Online]. Available: https://www.pnas.org/content/116/12/5451]

[9] D. L. Donoho, A. Maleki, and A. Montanari, "Message-passing algorithms for compressed sensing," Proceedings of the National Academy of Sciences, vol. 106, no. 45, pp. 18914-18919, Nov. 2009.

[10] M. Bayati and A. Montanari, "The dynamics of message passing on dense graphs, with applications to compressed sensing," IEEE Trans. Inform. Theory, vol. 57, no. 2, pp. 764-785, Feb. 2011.

[11] G. Reeves and M. Gastpar, "The sampling rate-distortion tradeoff for sparsity pattern recovery in compressed sensing," IEEE Trans. Inf. Theor., vol. 58, no. 5, pp. 3065-3092, May 2012. [Online]. Available: http://dx.doi.org/10.1109/TIT.2012.2184848

\section{A Properties of RS and AMP Formulas under finite entropy}

This section describes some properties of the potential function $\mathcal{F}(s)$ defined in (11), such as its minimum value $\mathcal{F}^{*}$, the upper and lower minimizers $\left(\bar{s}^{*}, \underline{s}^{*}\right)$, and the smallest stationary point $s^{\mathrm{AMP}}$, as a function of the problem parameters $(\delta, \mathrm{snr}, \pi)$. Throughout, we make the additional assumption that $\pi$ is a discrete distribution with finite entropy $H=H(\pi)$.

Lemma 9. The mutual information function $I(s)$ of the single-letter channel under input distribution $\pi$ defined in (7) satisfies

$$
\min \left\{\frac{s}{2}, H\right\}-L \leq I(s) \leq \min \left\{\frac{s}{2}, H\right\}
$$

for all $s \in[0, \infty)$, where

$$
L:=H-I(2 H) .
$$

Proof. Define $A(s):=s / 2-I(s)$. By the I-MMSE relation and the assumption of the unit variance, the derivative $A^{\prime}(s)=(1-M(s)) / 2$ is nonnegative, and thus $0 \leq s \leq 2 H$ implies that $A(0) \leq A(s) \leq A(2 H)$. Noting that $A(0)=0$ and $A(2 H)=L$ yields $0 \leq s / 2-I(s) \leq L$ for all $s \in[0,2 H]$. Meanwhile, the mutual information also satisfies the upper bound $I(s) \leq H$ for all $s$. Finally, because $I(s)$ is non-decreasing, $s \geq 2 H$ implies that $I(s) \geq I(2 H)=H-L$. Combining these inequalities gives the stated result.

\section{A.1 Minimizer of potential function}

We now consider upper and lower bounds on the minimizers of the potential function $\mathcal{F}(s)$ defined in (11). The basic idea behind our approach is to use the following simple relations for the minimizers of $\mathcal{F}(s)$ :

$$
\begin{gathered}
\min _{s \in(0, t]} \mathcal{F}(s)>\min _{s \in(0, \infty)} \mathcal{F}(s) \quad \Longrightarrow \quad \underline{s}^{*}>t \\
\min _{s \in(0, \infty)} \mathcal{F}(s)<\min _{s \in[t, \infty)} \mathcal{F}(s) \quad \Longrightarrow \quad \bar{s}^{*}<t .
\end{gathered}
$$

Lemma 10. For any $t \in[2 H, \infty)$,

$$
\delta>\frac{t+2 L}{\log (1+\mathrm{snr})} \quad \Longrightarrow \quad \underline{s}^{*}>t .
$$


Proof. Observe that $\phi(x)$ is convex and thus $\phi(y) \geq \phi(x)+(y-x) \phi^{\prime}(x)$ for all $x, y \in(0, \infty)$. Noting that $\phi^{\prime}(x)=1-1 / x$ and evaluating with $y=s /(\delta$ snr $)$ and $x=1 /(1+\operatorname{snr})$ leads to

$$
\phi\left(\frac{s}{\delta \mathrm{snr}}\right) \geq \log (1+\mathrm{snr})-\frac{s}{\delta} .
$$

Using this inequality to lower bound the potential function, we have

$$
\begin{aligned}
\min _{s \in[0, t)} \mathcal{F}(s) & \geq \min _{s \in[0, t)}\left\{I(s)+\frac{\delta}{2} \log (1+\mathrm{snr})-\frac{s}{2}\right\} \\
& \geq H-L+\frac{\delta}{2} \log (1+\mathrm{snr})-\frac{t}{2}>H,
\end{aligned}
$$

where the second step follows from Lemma 9 and the assumption $t \geq 2 H$, and the last step follows from the assumption on $\delta$. Meanwhile, using the upper bound $I(s) \leq H$, we have

$$
\min _{s \in(0, \infty)} \mathcal{F}(s) \leq \min _{s \in(0, \infty)}\left\{H+\frac{\delta}{2} \phi\left(\frac{s}{\delta \mathrm{snr}}\right)\right\}=H .
$$

Thus, we can conclude that the minimum is not attained in the interval $(0, t]$.

Lemma 11. For any $t \in(0,2 H]$,

$$
\delta<\frac{t-2 L}{\log (1+\mathrm{snr})} \quad \Longrightarrow \quad \bar{s}^{*}<t .
$$

Proof. Noting that $\phi(x) \geq 0$, we have

$$
\min _{s \in[t, \infty)} \mathcal{F}(s) \geq \min _{s \in[t, \infty)} I(s) \geq I(t) \geq \frac{t}{2}-L>\frac{\delta}{2} \log (1+\mathrm{snr}),
$$

where the third step follows from Lemma 9 for $s=t$ and the assumption $t \leq 2 H$, and the last step follows from the assumption on $\delta$. Meanwhile, using the upper bound $I(s) \leq s / 2$, we have

$$
\min _{s \in(0, \infty)} \mathcal{F}(s) \leq \min _{s \in(0, \infty]}\left\{\frac{s}{2}+\frac{\delta}{2} \phi\left(\frac{s}{\delta \text { snr }}\right)\right\}=\frac{\delta}{2} \log (1+\text { snr }) .
$$

Thus, we can conclude that the minimum is not attained in the interval $[t, \infty)$.

\section{A.2 Smallest stationary point of the potential function}

Lemma 12. For any $t \in(0, \infty)$,

$$
\begin{array}{lll}
\delta<\sup _{s \in(0, t]} s\left(M(s)+\frac{1}{\mathrm{snr}}\right) & \Longrightarrow & s^{\mathrm{AMP}}<t \\
\delta>\sup _{s \in(0, t]} s\left(M(s)+\frac{1}{\mathrm{snr}}\right) & \Longrightarrow & s^{\mathrm{AMP}}>t .
\end{array}
$$

Proof. By differentiation and the I-MMSE relation, one finds that every stationary point of $\mathcal{F}(s)$ satisfies the fixed-point equation $M(s)-\delta / s+1 / \mathrm{snr}=0$. Rearranging and solving for $\delta$, we see that $s$ is a stationary point if and only if $\delta_{\mathrm{FP}}(s)=\delta$ where

$$
\delta_{\mathrm{FP}}(s):=s\left(M(s)+\frac{1}{\mathrm{snr}}\right) .
$$

The function $\delta_{\mathrm{FP}}(s)$ is continuous with $\delta_{\mathrm{FP}}(0)=0$. Therefore, if $\delta<\sup _{s \in(0, t]} \delta_{\mathrm{FP}}(s)$ then the equation $\delta_{\mathrm{FP}}(s)=\delta$ has at least one solution on $[0, t)$, and this implies that $s^{\mathrm{AMP}}<t$. Conversely, if $\delta>$ $\sup _{s \in(0, t]} \delta_{\mathrm{FP}}(s)$ then there is no solution on $[0, t)$ and this implies that $s^{\mathrm{AMP}}>t$. 


\section{B Proofs of Main Results}

\section{B.1 Proof of Theorem 4}

First we show that Assumption 1 implies that $L_{\epsilon} / H_{\epsilon} \rightarrow 0$, where we recall that $L_{\epsilon}=H_{\epsilon}-I_{\epsilon}\left(2 H_{\epsilon}\right)$. To see why, observe that

$$
L_{\epsilon}=\frac{1}{2} \int_{0}^{2 H_{\epsilon}}(1-M(s)) \mathrm{d} s=H_{\epsilon} \int_{0}^{1}\left(1-M\left(2 H_{\epsilon} t\right)\right) \mathrm{d} t .
$$

For any $\eta \in(0,1)$, the integral on the right can be upper bounded as

$$
\int_{0}^{1}\left(1-M\left(2 H_{\epsilon} t\right)\right) \mathrm{d} t \leq \int_{0}^{1-\eta}\left(1-M\left(2 H_{\epsilon} t\right)\right) \mathrm{d} t+\eta .
$$

By Assumption 1, the first term on the right-hand side converges to zero in the small- $\epsilon$ limit. Noting that $\eta$ can be chosen arbitrarily small establishes that $L_{\epsilon} / H_{\epsilon} \rightarrow 0$.

We are now ready to consider the case $r \in(0,1)$. Fix $\eta$ such that $r<1-\eta<1$ and let $t_{\epsilon}=(1-\eta / 2) 2 H_{\epsilon}$. For all $\epsilon$ sufficiently small, we have $\eta H_{\epsilon}>2 L_{\epsilon}$ and thus $t_{\epsilon}-2 L_{\epsilon} \geq(1-\eta) 2 H_{\epsilon}>r 2 H_{\epsilon}$. Under the assumed scaling in (21), this means that $\delta_{\epsilon} \leq\left(t_{\epsilon}-2 L_{\epsilon}\right) / \log \left(1+\mathrm{snr}_{\epsilon}\right)$ for all $\epsilon$ small enough. By Lemma 11, this implies that $\bar{s}^{*}<(1-\eta / 2) 2 H_{\epsilon}$ and by Assumption 1 it follows that $M_{\epsilon}\left(\bar{s}_{\epsilon}^{*}\right) \rightarrow 1$. The case $r \in(1, \infty)$ follows from a similar argument and is omitted.

\section{B.2 Proof of Theorem 6}

Consider the case $r \in(0,1)$. Fix $\eta$ such that $r<(1-\eta)^{2}<1$ and let $t_{\epsilon}=(1-\eta) 2 H_{\epsilon}$. By (20), for all $\epsilon$ sufficiently small, we have $M_{\epsilon}(s) \geq 1-\eta$ for all $s \in\left[0, t_{\epsilon}\right]$, and thus

$$
\begin{aligned}
\sup _{s \in\left[0, t_{\epsilon}\right]} s\left(M_{\epsilon}(s)+\frac{1}{\operatorname{snr}_{\epsilon}}\right) & \geq \sup _{s \in\left[0, t_{\epsilon}\right]} s\left(1-\eta+\frac{1}{\operatorname{snr}_{\epsilon}}\right) \\
& =(1-\eta) 2 H_{\epsilon}\left(1-\eta+\frac{1}{\text { snr }_{\epsilon}}\right) \\
& >(1-\eta)^{2} 2 H_{\epsilon}\left(1+\frac{1}{\operatorname{snr}_{\epsilon}}\right) .
\end{aligned}
$$

The scaling (25) combined with the assumption $r<(1-\eta)^{2}$ means that, for all $\epsilon$ small enough,

$$
\delta_{\epsilon}<\sup _{s \in\left[0, t_{\epsilon}\right]} s\left(M_{\epsilon}(s)+\frac{1}{\operatorname{snr}_{\epsilon}}\right) .
$$

By Lemma 12, this implies that $s_{\epsilon}^{\text {AMP }}<t_{\epsilon}$ and by (20), this means that $M_{\epsilon}\left(s_{\epsilon}^{\text {AMP }}\right) \rightarrow 1$. The case $r \in(1, \infty)$ follows from a similar argument and is omitted. 